International Journal of Engineering \& Technology, $7(4.10)(2018) 589-592$
International Journal of Engineering \& Technology
SPC
Website: www.sciencepubco.com/index.php/IJET
Research paper

\title{
The Split Distance 2 Domination in Graphs
}

\author{
A. Lakshmi ${ }^{1, *}$, K. Ameenal Bibi ${ }^{2}$, R. Jothilakshmi ${ }^{3}$ \\ ${ }^{1} P G$ and Research Department of Mathematics, D.K.M. College for women (Autonomous), Vellore - 632 001, India \\ ${ }^{2}$ Department of Mathematics, D.K.M. College for women (Autonomous), Vellore-632 001, India \\ ${ }^{3}$ Department of Mathematics, MazharulUloom College, Ambur, India \\ *Corresponding author E-mail: lprabha24@gmail.com.
}

\begin{abstract}
A distance - 2 dominating set $\mathrm{D} \subseteq \mathrm{V}$ of a graph $\mathrm{G}$ is a split distance -2 dominating set if the induced sub graph $\langle\mathrm{V}$-D $\rangle$ is disconnected. The split distance -2 domination number $\gamma_{s \leq 2}(G)$ is the minimum cardinality of a split distance -2 dominating set. In this paper, we defined the notion of split distance - 2 domination in graph. We got many bounds on distance - 2 split domination number. Exact values of this new parameter are obtained for some standard graphs. Nordhaus - Gaddum type results are also obtained for this new parameter.
\end{abstract}

Key words: Dominating set, split dominating set, distance -2 dominating set, split distance -2 dominating set, split distance -2 domination Number.

\section{Introduction}

All graphs considered here are simple, finite, connected and undirected. Let $\mathrm{n}$ and $\mathrm{m}$ denote the order and size of a graph $\mathrm{G}$. We use the terminology of [11]. Let $\Delta(G)(\delta(G))$ denote the maximum (minimum) degree and $\lfloor x\rfloor(\lceil x\rceil)$ the greatest (least) integer less (greater) than or equal to $\mathrm{x}$. The independence number $\beta_{0}(\mathrm{G})$ is the maximum cardinality among the independent set of vertices of $G$. The lower independence number $i(G)$ is the minimum cardinality among the maximum independent set of vertices of $\mathrm{G}$. The vertex covering number $\alpha_{0}(\mathrm{G})$ is the minimum cardinality of vertex covering of $G$. The girth $g(G)$ of a graph $G$ is the length of the shortest cycle in G. The circumference $c(G)$ is the length of the longest cycle. The radius of $G$ is $\operatorname{rad}(G)=$ $\min \{\operatorname{ecc}(\mathrm{v}): \mathrm{v} \in V\}$ and $\operatorname{diam}(\mathrm{G})=\max \{\operatorname{ecc}(\mathrm{v}): \mathrm{v} \in V\}$, where $\operatorname{ecc}(\mathrm{v})=\max \{\operatorname{dis}(\mathrm{u}, \mathrm{v}): \mathrm{v} \in V\}$.

A nonempty subset $D \subseteq V(G)$ is said to be a dominating set of $\mathrm{G}$ if every vertex not in $\mathrm{D}$ is adjacent to at least one vertex in $\mathrm{D}$. A dominating set $\mathrm{D} \subseteq \mathrm{V}$ of a graph $\mathrm{G}$ is a split dominating set if the induced sub-graph $<\mathrm{V}$-D $>$ is disconnected. The split domination number $\gamma_{s}(G)$ is the minimum cardinality of a split dominating set. A set $\mathrm{D}$ of vertices in a graph $\mathrm{G}$ is a distance - 2 dominating set if every vertex in V-D is within distance - 2 of at least one

vertex in D. The distance - 2 domination number $\gamma_{\leq 2}(G)$ of $\mathrm{G}$ equals the minimum cardinality of a distance 2 - dominating set in G.

Kulli V.R. and Janakiram B. introduced the concept of split domination in graph in [13]. The purpose of this paper is to introduce the concept of split distance - 2 domination in graphs.

\section{Definition 1.1}

A distance - 2 dominating set $D \subseteq V$ of a graph $\mathrm{G}$ is a split distance - 2 dominating set if the induced sub graph $\langle\mathrm{V}-\mathrm{D}\rangle$ is disconnected. The split distance - 2 domination number $\gamma_{s \leq 2}(G)$ is the minimum cardinality of a minimal split distance - 2 dominating set.

The minimal distance - 2 dominating set in a graph $\mathrm{G}$ is a distance - 2 dominating set that contains no distance -2 dominating set as a proper subset.

The distance - 2 open neighborhood of a vertex $v \in V$ is the set, $N_{\leq 2}(v)$, of vertices within a distance of two from $(v)$.

Example: 1.2

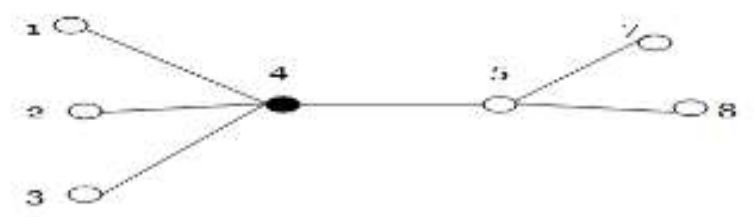

Figure.1

Here $D=\{4\}, \gamma_{s \leq 2}(G)=1$

\section{Common Graphs and Exact Values}

The split distance - 2 domination number $\gamma_{s \leq 2}(G)$ of some common graphs is given as an observation.

\subsection{Observation}

1. For any path $P_{n}$, for $n \geq 2$

2. For any cycle $C_{n}$, for $\mathrm{n} \geq 6$

$$
\gamma_{s \leq 2}\left(P_{n}\right)=\left\lceil\frac{n}{5}\right\rceil
$$

$$
\gamma_{s \leq 2}\left(C_{n}\right)=\left\lceil\frac{n+1}{5}\right\rceil
$$

3. For any complete graph $\mathrm{K}_{\mathrm{n}}$, for $\mathrm{n} \geq 3$

$$
\gamma_{s \leq 2}\left(K_{n}\right)=n-1
$$


4. For any star graph $\mathrm{K}_{1}, \mathrm{~m}$, for $\mathrm{m} \geq 1$

$$
\gamma_{s \leq 2}\left(K_{1, m}\right)=1
$$

5. For any complete bipartite graph $\mathrm{K}_{\mathrm{n}, \mathrm{m}}$, for $\mathrm{m} \geq \mathrm{n}$,

$$
\gamma_{s \leq 2}\left(K_{n, m}\right)=n
$$

\section{Bounds on the Split Distance -2 Domination Number $\gamma_{s \leq 2}(G)$}

\section{Theorem 3.1}

For any graph $\mathrm{G}, \gamma_{\leq 2}(G) \leq \gamma_{s \leq 2}(G)$.

Proof

Every split distance - 2 dominating set of $\mathrm{G}$ is a distance - 2 dominating set of G. We have, $\gamma_{\leq 2}(G) \leq \gamma_{s \leq 2}(G)$.

\section{Theorem 3.2}

For any graph $\mathrm{G}, \gamma_{s \leq 2}(G) \leq \gamma_{s}(G)$.

\section{Proof}

Every split dominating set of $\mathrm{G}$ is a split distance - 2 dominating set of G. Hence $\gamma_{s \leq 2}(G) \leq \gamma_{s}(G)$.

\section{Theorem 3.3}

Let $\mathrm{D}$ is a minimal split distance - 2 dominating set if and only if each vertex $v \in D$, satisfies at least one of the following conditions

(i) There exists a vertex $u \in V-D$, such that

Case (a): When D is connected, $N_{\leq 2}(u) \cap D=D$

Case (b): When D is disconnected, $N_{\leq 2}(u) \cap D=\{v\}$

(ii) $v$ is an isolated vertex in $\langle\mathrm{D}\rangle$

(iii) $\{(V-D) \cup\{v\}\}$ is connected.

\section{Proof}

Suppose D is a minimal split distance - 2 dominating set. On the contrary, there exists a vertex $v \in D$, such that $v$ does not hold any of the above conditions. Then by conditions (i) and (ii), $D_{1}=D-$ $\{v\}$ isa distance -2 dominating set of G. Also by (iii), $\left\langle\mathrm{V}-\mathrm{D}_{1}\right\rangle$ is disconnected. Hence $D_{1}$ is a split distance - 2 dominating set of $G$, which is a contradiction. Therefore, the condition is necessary.

Sufficiency follows from the given conditions.

\section{Theorem 3.4}

For any graph $\mathrm{G}$ with a pendant vertex

$\gamma_{\leq 2}(G)=\gamma_{s \leq 2}(G)$,

Furthermore, there exists a split distance - 2 dominating set of $\mathrm{G}$ containing all vertices within the distance -2 pendant vertices.

\section{Note: $\mathbf{3 . 5}$}

For any graph $\mathrm{G}$, we observe $\gamma_{\leq 2}(G) \leq \gamma(G)$.

Theorem 3.6 (Kulli and Janakiram, 1997)

For any graph $\mathrm{G}, \gamma_{s}(G) \leq \alpha_{0}(G)$.

\section{Theorem 3.7}

For any graph $\mathrm{G}, \gamma_{s \leq 2}(G) \leq \alpha_{0}(G)$.

\section{Proof}

Since $\gamma_{s \leq 2}(G) \leq \gamma_{s}(G)$ and $\gamma_{s}(G) \leq \alpha_{0}(G)$ [By Theorem 3.6] We have $\gamma_{s \leq 2}(G) \leq \alpha_{0}(G)$.

\section{Theorem 3.8}

For any graph $\mathrm{G}, \gamma_{\leq 2}(G)+\gamma_{s \leq 2}(G) \leq n$.

\section{Proof}

Since $\gamma(G) \leq \beta_{0}(G), \gamma_{\leq 2}(G) \leq \gamma(G)$ and $\gamma_{s}(G) \leq \alpha_{0}(G)$ [By

Theorem 3.6]

Thus $\gamma_{\leq 2}(G)+\gamma_{s \leq 2}(G) \leq \alpha_{0}(G)+\leq \beta_{0}(G)$,
We have $\gamma_{\leq 2}(G)+\gamma_{s \leq 2}(G) \leq n$.

The sharpness of this equality can be seen with the path $\mathrm{P}_{2}$ and cycle $\mathrm{C}_{3}$

\section{Theorem 3.9}

For any graph $\mathrm{G},(G)+\gamma_{s \leq 2}(G) \leq n$.

Proof

Since $(G) \leq \beta_{0}(G)$, and $\gamma_{s}(G) \leq \alpha_{0}(G)$ [By Theorem 3.6]

Thus $i(G)+\gamma_{s \leq 2}(G) \leq \alpha_{0}(\mathrm{G})+\beta_{0}(\mathrm{G})$

We have $i(G)+\gamma_{s \leq 2}(G) \leq n$.

The sharpness of this equality can be seen with the path $\mathrm{P}_{2}$, cycle $\mathrm{C}_{3}, \mathrm{C}_{4}$.

Theorem3.10 (Kulli and Janakiram, 1997)

For any graph $\mathrm{G}, \gamma_{s}(G) \leq \frac{n \cdot \Delta(G)}{(\Delta(G)+1)}$.

Theorem 3.11 (Kulli and Janakiram, 1997)

For any graph $\mathrm{G}, \gamma_{s \leq 2}(G) \leq \frac{n \cdot \Delta(G)}{(\Delta(G)+1)}$.

\section{Proof}

Since $\gamma_{s \leq 2}(G) \leq \gamma_{s}(G)$ and $\gamma_{s}(G) \leq \frac{n . \Delta(G)}{(\Delta(G)+1)}[$ By Theorem 3.10] We have, $\gamma_{s \leq 2}(G) \leq \frac{n \cdot \Delta(G)}{(\Delta(G)+1)}$.

Theorem 3.12

For any graph $\mathrm{G}, \gamma_{s \leq 2}(G)=\gamma_{s}(G)$ if and only if $\mathrm{G}$ is a wheel $\mathrm{W}_{\mathrm{n}}$

Theorem 3.13

For any graph G, $\gamma_{s \leq 2}(G)=\gamma_{s}(G)=\gamma_{\leq 2}(G)=\gamma(G)$ if and only if $\mathrm{G}$ is a star $\mathrm{K}_{1, \mathrm{~m}}$, for $\mathrm{m}>1$.

\section{Theorem 3.14}

For any graph $\mathrm{G}, \gamma_{s \leq 2}(G)=\gamma_{s}(G)=\gamma(G)$ if and only if $\mathrm{G}$ is a friendship graph $\mathrm{F}_{\mathrm{n}}$.

\section{Theorem 3.15}

For any graph $\mathrm{G}, \gamma_{s \leq 2}(G)=\gamma_{s}(G)$ if and only if $\mathrm{G}$ is a bipartite graph $\mathrm{K}_{\mathrm{n}, \mathrm{m}}$, for $\mathrm{n}<\mathrm{m}$.

\section{Theorem 3.16}

For any graph $\mathrm{G}, \gamma_{s \leq 2}(G)=\mathrm{p}-\Delta(\mathrm{G})$ if and only if $\mathrm{G}$ is a star graph $\mathrm{K}_{1, \mathrm{~m}}$, for $\mathrm{m}>1$, where $p=1+m$ is number of vertices.

\section{Theorem 3.17}

For any graph $\mathrm{G}$, which is not a tree then $\gamma_{s \leq 2}(G) \leq c(G)$ where $c(G)$ is circumference of a graph $G$.

\section{Theorem 3.18}

For any graph $\mathrm{G}$, which is not a tree then $\gamma_{s \leq 2}(G) \leq g(G)$ where $g(G)$ is girth of a graph $G$.

\section{Theorem 3.19}

For any graph $\mathrm{G}, \gamma_{s \leq 2}(G) \leq \mathrm{n}-\Delta(\mathrm{G})$.

Note 3.20

For any graph $\mathrm{G}, \gamma_{s \leq 2}(G) \leq \Delta(\mathrm{G})$.

\section{Theorem 3.21}

For any tree $\mathrm{T}_{\mathrm{n}}, \gamma_{s \leq 2}(G) \leq p$ where $\mathrm{p}$ is number of end vertices.

Note 3.22

(i) For any tree $\mathrm{T}_{\mathrm{n}}$, which is a star graph $\gamma_{s \leq 2}(G)=n-p$ where $\mathrm{n}$ is number of vertices and $\mathrm{p}$ is number of end vertices.

(ii) $\gamma_{s \leq 2}\left(T_{n}\right)=\gamma_{\leq 2}\left(T_{n}\right)$. 


\section{Theorem 3.23}

If $\mathrm{H}$ is a connected spanning sub graph of $\mathrm{G}$, then $\gamma_{s \leq 2}(G) \leq$ $\gamma_{s \leq 2}(H)$.

\section{Theorem 3.24}

Let $\mathrm{G}$ be any connected graph of order greater than 3, then $\gamma_{s \leq 2}(G) \leq n-1$, where $\mathrm{n}$ is the number of vertices.

\section{Nordhas - Gaddum Type results}

\section{Theorem 3.25}

Let $\mathrm{G}$ be a graph such that both $\mathrm{G}$ and $\bar{G}$ have no isolates. Then,

(i) $\gamma_{s \leq 2}(G)+\gamma_{s \leq 2}(\bar{G}) \leq 2(n-1)$

(ii) $\gamma_{s \leq 2}(G)+\gamma_{s \leq 2}(\bar{G}) \leq(n-1)^{2}$

\section{Lemma 3.26}

Fork $\geq 1$, every connected graph $\mathrm{G}$ has a spanning tree $\mathrm{T}$ such that $\gamma_{k}(G)=\gamma_{k}(H)$ in [23]

\section{Lemma 3.27}

Fork $\geq 1$, every connected graph $\mathrm{G}$ has a spanning tree $\mathrm{T}$ such that $\gamma_{s \leq 2}(G)=\gamma_{s \leq 2}(H)$

\section{Proof}

Since $\gamma_{\leq 2}(G) \leq \gamma_{s \leq 2}(G)$ and $\gamma_{\leq 2}(T)=\gamma_{\leq 2}(G)$ [By Lemma 3.26].

We have $\gamma_{s \leq 2}(G)=\gamma_{s \leq 2}(H)$.

\section{Theorem 3.28}

For $k \geq 1$, if $\mathrm{G}$ is a connected graph with diameter $\mathrm{d}$, then $\gamma_{k}(G) \geq \frac{d+1}{2 k+1}$ in [23].

\section{Theorem 3.29}

For any graph $\mathrm{G}$ is a connected graph with diameter $\mathrm{d}$, then $\gamma_{s \leq 2}(G) \geq \frac{d+1}{2 k+1}$

\section{Proof}

Since $\gamma_{\leq 2}(G) \leq \gamma_{s \leq 2}(G)$ and $\gamma_{\leq 2}(G) \geq \frac{d+1}{2 k+1}$ [By Theorem 3.28]. Thus we have, $\gamma_{s \leq 2}(G) \geq \frac{d+1}{2 k+1}$.

\section{Theorem 3.30}

If $\mathrm{G}=\mathrm{P}_{\mathrm{n}}$ where $n \equiv 0 \bmod (2 k+1)$, then $\gamma_{k}(G)=\frac{\operatorname{diam}(G)+1}{2 k+1}$ in [23]

\section{Theorem 3.31}

If $\mathrm{G}=\mathrm{P}_{\mathrm{n}}$ wheren $\equiv 0 \bmod (2 k+1)$, then $\gamma_{s \leq 2}(G)=\frac{\operatorname{diam}(G)+1}{2 k+1}$. Proof

Since $\gamma_{\leq 2}(G) \leq \gamma_{s \leq 2}(G)$ and $\gamma_{\leq 2}(G)=\frac{\operatorname{diam}(G)+1}{2 k+1}[$ By Theorem 3.30]

We have $\gamma_{s \leq 2}(G) \geq \frac{\operatorname{diam}(G)+1}{2 k+1}$

\section{Theorem 3.32}

For any graph $\mathrm{G}$ is a connected graph with radius $\mathrm{r}$ then $b \gamma_{s \leq 2}(G) \geq \frac{2 r}{2 k+1}$ in [23].

\section{Theorem 3.33}

For any graph $\mathrm{G}$ is a connected graph with radius $\mathrm{r}$, then $\gamma_{k}(G) \geq$ $\frac{2 r}{2 k+1}$.

\section{Proof}

Since $\gamma_{\leq 2}(G) \leq \gamma_{s \leq 2}(G)$ and $\gamma_{\leq 2}(G) \geq \frac{2 r}{2 k+1}$ [By Theorem 3.32] We have $\gamma_{s \leq 2}(G) \geq \frac{2 r}{2 k+1}$

\section{Theorem 3.34}

For any graph $\mathrm{G}$ is a connected graph with girth g, then $\gamma_{k}(G) \geq$ $\frac{g}{2 k+1}$ in [23].

Theorem 3.35

For any graph $\mathrm{G}$ is a connected graph with girth $\mathrm{g}$, then $\gamma_{s \leq 2}(G) \geq$ $\frac{g}{2 k+1}$

\section{Proof}

Since $\gamma_{\leq 2}(G) \leq \gamma_{s \leq 2}(G)$ and $\gamma_{\leq 2}(G) \geq \frac{g}{2 k+1}$ [By Theorem 3.34] We have $\gamma_{s \leq 2}(G) \geq \frac{g}{2 k+1}$.

\section{Conclusion}

In general, the concept of dominating sets in graph theory finds wide applications in different types of communication networks. Identification of a dominating set, which is fault tolerant, will be useful in the central location theory.

Thus in this paper, we defined the notion of split distance - 2 domination in graphs. We obtained many bounds on distance - 2 split domination number. Exact values of this new parameter are obtained for some standard graphs. Nordhaus - Gaddum type results are also obtained for this new parameter. They play very vital role in Coding theory, Computer science, Operations Research, Switching Circuits, and Signal Processing Electrical Networks etc.

\section{References}

[1] AmeenalBibi, K. and Selvakumar, R (2010), The Inverse split and non-split domination numbers in graph. International Journal of Computer Applications 8(7), pp. 21-29.

[2] AmeenalBibi, K. and Selvakumar, R (2010), The Inverse strong nonsplit r-domination number of a graph. International Journal of Engineering, Science and Technology, Vol.2, No.1, pp.127-133

[3] Cockayne, E.J. Dawes, R.M. and Hedetniemi, S.T. (1980). Total domination in graphs. Networks, vol.10, pp.211-219.

[4] E.J.Cockayne, S.T.Hedetniemi (1977), Towards a Theory of Domination in Graphs, Networks. vol.7, pp.247-261.

[5] Domke G.S., Dunbar J.E. and Markus, L.R (2004), The Inverse domination number of a graph, Ars. Combin 72, pp.149-160.

[6] O.Favaron and D.Kratsch (1991) Ratios of domination parameters, Advances in graph theory, Viswa International Publication, Gulbarga, pp.173-182.

[7] P. Fraisse(1988), A note on distance dominating cycles. Discrete Math. 71, pp.89-92.

[8] A. Hansberg, D. Meierling, and L. Volkmann (2007), Distance domination and distance irredundance in graphs. Electronic J.Combin. 14, \#R35.

[9] Harary. F (1969) Graph Theory, Addison - Wesley Reading Mars.

[10] Haynes, T.W., Hedetniemi .S.T. and Slater.P.J (1998), Domination in Graphs: Advanced Topics, Marcel Dekker Inc. New York, U.S.A.

[11] Haynes, T.W., Hedetniemi .S.T. and Slater.P.J (1998)b, Fundamentals of domination in Graphs, Marcel Dekkel Inc. New York, U.S.A.

[12] M.A. Henning, O.R. Oellermann and H.C. Swart (1991), Bounds on distance domination parameters. J.Combin. Inform. System Sci 16, pp.11-18.

[13] V.R.Kulli and B.Janakiram (1997), The split domination number of a graph. Graph Theory Notes of New York, New York Academy of Sciences, XXXII, pp.16-19.

[14] V.R.Kulli and B.Janakiram (2006), The strong split domination number of a graph. Acta Ciencia Indica, 32M, pp.715-720.

[15] V.R.Kulli and B.Janakiram (2000), The non-split domination number of a graph. Indian J. Pure Appl. Math, pp.545-550

[16] V.R.Kulli and B.Janakiram(2003), The strong non-split domination number of a graph. Internat. J. Management Systems,19, pp.145156.

[17] Kulli, V.R and Sigarkanti, S.C (1991), Inverse dominating in graphs. National Academy Science Letters, 15. 
[18] V.R. Kulli (2010), Theory of domination in graphs. Vishwa International Publications, Gulbarga, India.

[19] V. R. Kulli (2012), Advances in domination theory Vishwa International Publications, Gulbarga, India.

[20] D. Meierling and L.Volkmann (2005), A lower bound for the distance k-domination number of trees, Result. Math. 47, pp.335339.

[21] Nordhaus, E.A and Gaddam, J.W (1956). On complementary graphs. Amer. Math. Monthly, Vol.63, pp.175-177.

[22] Ore, O.(1962), Theory of Graphs. American Mathematical Society colloq. Publ., Providence, R1, 38.

[23] Randy Davila, Caleb Fast, Michael A. Henning and Franklin Kenter (2015), Lower bounds on the distance domination number of a Graph. arXiv:1507.08745v1 [math.co] 31.

[24] F. Tian and J.M.Xu (2009), A note on distance domination numbers of graphs. Australasian j. Combin. 43, pp.181-190. 